\title{
Phase behavior of ternary polymer brushes
}

Chester K. Simocko, Amalie L. Frischknecht, Dale L. Huber*

Center for Integrated Nanotechnologies, Sandia National Laboratories, Albuquerque NM 87185

\section{Supporting Information}

Synthesis and Characterization of Ternary Polymer Brushes. Scheme SI1 depicts the synthesis of ternary polymer brushes. A thin, uniform layer of aminopropyltriethyoxysilane (APTES) is applied to the surface via vapor deposition. The aminated surface is then reacted with acid chloride of 4,4'-azobis(4-cyanovaleric acid) (ACVA), synthesized via previous reported procedure, to create a uniform surface with a thickness of usually $3-5 \mathrm{~nm}$. Free radical surface initiated polymerizations are done in a monomer solution containing unbound initiator as has been previously reported. ${ }^{1}$ ACVA has a reported half-life of $\approx 10 \mathrm{~h}$ at $65^{\circ} \mathrm{C}$ so the volume fraction of each polymer can be controlled by the length of each polymerization (PS, PMMA, or P4VP); shorter polymerization times lead to lower volume fractions while longer reaction times lead to larger volume fractions. After the first polymerization is complete there is a population of unreacted initiator still on the surface which allows for subsequent polymerizations to take place. Following each growth of polymer the wafer is washed, the dry brush thickness is measured via ellipsometry, and the molecular weight of the ungrafted polymer is measured by gel permeation chromatography. The ternary polymer brush is then annealed in chloroform vapor for $24 \mathrm{~h}$ followed by thermal annealing under vacuum at $125^{\circ} \mathrm{C}$ for $24 \mathrm{~h}$. A detailed procedure is described below.

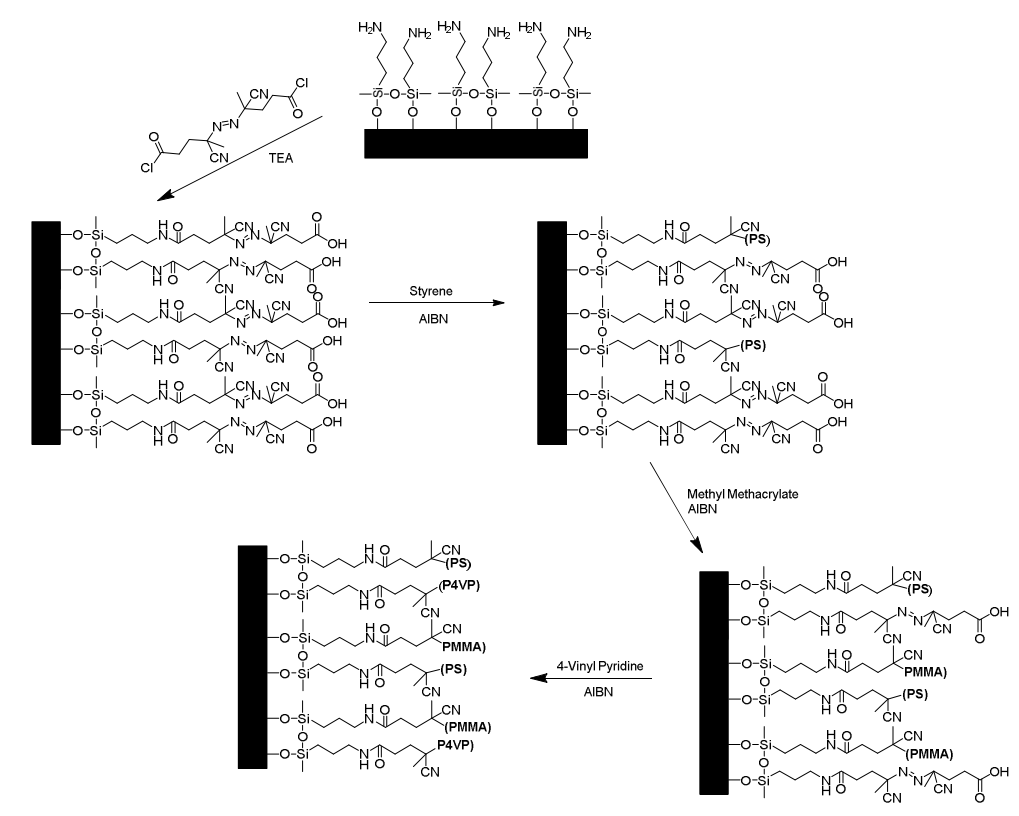

Scheme SI1. Synthesis of ternary polymer brushes 


\section{Materials}

Silicon wafers (Silicon Quest International) were test grade P-type, CZ silicon doped with boron. 2,2'-Azobis(2-methylpropionitrile) (AIBN, Aldrich, 98\%) and 4,4'-azobis(4-cyanovaleric acid) (ACVA, Aldrich, $\geq 98 \%$ ) were recrystallized twice from methanol. Methyl methacrylate (Aldrich, 99\%), styrene (Aldrich, $\geq 99 \%$ ), and 4-vinyl pyridine were distilled under reduced pressure to remove inhibitor. Triethylamine (Alfa Aesar, $\geq 99 \%$ ) was distilled from calcium hydride and stored over $3 \AA$ molecular sieves. All other chemicals were used as received.

\section{Methods}

Growth from the surface was measured using a Nanofilm EP3 imaging ellipsometer operating with an internal solid-state laser at $532 \mathrm{~nm}$ and angle of incidence of $70^{\circ}$. Results were obtained from three different spots on the wafer with three measurements per spot. The refractive indices used were 1.49 for PMMA, 1.59 for PS, and 1.55 for P4VP. The refractive indices for APMDES and ACVA were taken as 1.43 and 1.45 , respectively.

The phase and height morphologies of the polymer brushes were characterized by an Asylum MFP3D atomic force microscope (AFM). Tapping mode images were acquired with an aluminum coated silicon cantilever (Asylum Research) with a resonance frequency of $\sim 70 \mathrm{kHz}$, a force constant of $\sim 2 \mathrm{~N} / \mathrm{m}$, and a silicon with the tip radius of less than $9 \mathrm{~nm}$. Gwyddion and ImageJ were used for image analysis.

Polymer molecular weight and polydispersity were measured by gel permeation chromatography (GPC) against polystyrene standards using THF as the mobile phase for PS and PMMA. The columns were Waters Styragel HR4 followed by HR4E operated at room temperature with a flow rate of $1 \mathrm{~mL} / \mathrm{min}$. Molecular weights and polydispersity for P4VP were measured against poly (2vinyl pyridine) standards using DMF as the mobile phase and a Waters Styragel HR4E. All polymers were detected using a refractive index detector.

\section{Amination of a Silicon Surface}

A 3in. silicon wafer was washed with acetone and water then the surface was cleaned in a piranha solution $\left(7 / 3 \mathrm{v} / \mathrm{v}\right.$, Conc. $\left.\mathrm{H}_{2} \mathrm{SO}_{4} / 30 \% \mathrm{H}_{2} \mathrm{O}_{2}\right)$ at $80^{\circ} \mathrm{C}$ for 1 hour. The wafer was rinsed with water and dried then exposed to UV/ozone for 30 minutes. The surface of the wafer was hydrated with water then heated to $110^{\circ} \mathrm{C}$ for 5 minutes. The wafer is then suspended (smooth side down) over

a vial containing $2 \mathrm{~mL}$ of APTES and the chamber was evacuated to a static vacuum of 100mTorr for 16 hours. The wafer was annealed for 30 minutes at $110^{\circ} \mathrm{C}$ under vacuum. The wafer was washed with acetone, isopropanol, and water to remove unbound silane then dried under nitrogen.

\section{Initiator Attachment}

The acyl chloride of ACVA was synthesized and attached to the aminated silicon surface via previously published procedures. ${ }^{1}$

\section{Example of Surface-Initiated Polymerization}

All polymerizations were conducted in anhydrous 1,4 dioxane with AIBN, ACVA functionalized silicon wafer, and desired monomer in the following ratio: 
Table SI1. Experimental conditions for brush growth

\begin{tabular}{|c|c|c|c|}
\hline Monomer & $\begin{array}{l}\text { Dioxane } \\
\text { (mL:mol) }\end{array}$ & $\begin{array}{c}\text { AIBN } \\
(\mathrm{mg}: m m o l)\end{array}$ & $\begin{array}{l}\text { Monomer } \\
\text { Volume } \\
(\mathrm{mL}: \mathrm{mol})\end{array}$ \\
\hline Styrene & $\begin{array}{l}4.0 \\
.047\end{array}$ & $\begin{array}{l}3.0 \\
.018\end{array}$ & $\begin{array}{c}6.0 \\
.052\end{array}$ \\
\hline MMA & $\begin{array}{l}6.0 \\
.070 \\
\end{array}$ & $\begin{array}{l}5.0 \\
.030\end{array}$ & $\begin{array}{c}3.0 \\
.028 \\
\end{array}$ \\
\hline $4 \mathrm{VP}$ & $\begin{array}{l}4.0 \\
.047\end{array}$ & $\begin{array}{l}4.0 \\
.024\end{array}$ & $\begin{array}{l}6.0 \\
.056\end{array}$ \\
\hline
\end{tabular}

The solution underwent three cycles of freeze pump thawing to remove any dissolved oxygen and was then transferred into a sealed vial containing the silicon wafer. The polymerization was performed at $65^{\circ} \mathrm{C}$ for $2,4,6,8,10,14$, or 18 hours depending on desired polymer volume fraction. The free polymer was then precipitated into methanol (PS, PMMA) or hexanes (P4VP) to analyze via GPC. The wafer was then washed overnight with $\mathrm{CH}_{2} \mathrm{Cl}_{2}$ in a Soxhlet extractor. These steps were then repeated using the remaining monomer. The wafer was then solvent vapor annealed in chloroform followed by thermal annealing at $125^{\circ} \mathrm{C}$ under vacuum as previously reported. ${ }^{1}$ 
Table SI2. Characterization of PS/PMMA/P4VP ternary polymer brushes.

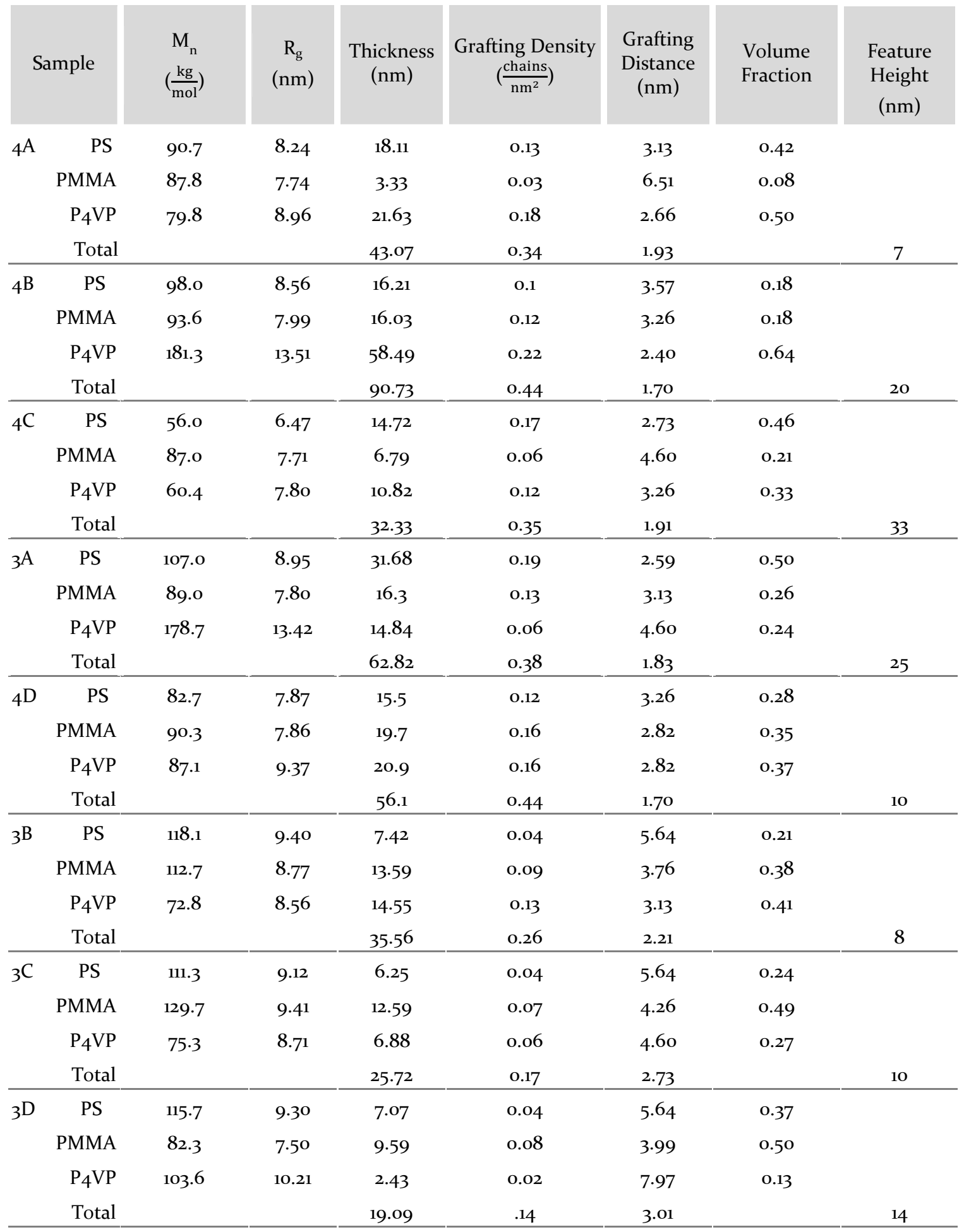


Table Calculations: The radius of gyration $R_{g}$ for PS and PMMA is calculated from equation (1)

(1) $R_{g}=\frac{r_{0}}{\sqrt{6}}$

The polymer end-to-end distance $\left(r_{0}\right)$ is calculated from equation (2) where $k=.0670 \mathrm{~nm}$ for PS and $.0640 \mathrm{~nm}$ for PMMA and $M_{n}$ is the average number molecular weight. ${ }^{1,2}$

(2) $r_{0}=k M_{n}{ }^{1 / 2}$

The radius of gyration for P4VP was calculated using equation (3) where $N$ is the degree of polymerization and $b$ is the average statistical segment length, $0.797 \mathrm{~nm}$ for P4VP. ${ }^{3}$

(3) $R_{g}=b(N / 6)^{1 / 2}$

The grafting density $(D)$ is calculated from equation (4) where $t$ is the brush thickness, $N_{a}$ is Avogadro's number and $\rho$ is the polymer density $\left(1.05 \mathrm{~g} / \mathrm{cm}^{3}\right.$ for PS, $1.19 \mathrm{~g} / \mathrm{cm}^{3}$ for PMMA and $1.11 \mathrm{~g} / \mathrm{cm}^{3}$ for P4VP). ${ }^{2,4}$

(4) $D=\frac{\rho t N_{a}}{M_{n} \times 10^{21}}$

The average distance between grafting points $(l)$ is calculated from the equation $l=2 /(\pi D)^{1 / 2} \cdot{ }^{4}$ Finally, the feature height is the difference in height between the lowest point in the sample (generally in the continuous phase) and the highest points.

Figure SI1 shows an AFM phase image of Sample 4B, to demonstrate how the AFM data looks before false color is applied. There are clearly three different domains in the image. Based on the $\mathrm{P} 4 \mathrm{VP}$ volume fraction of 0.64 , it must be the continuous domain and so in Fig $4 \mathrm{~B}$ in the main text, the continuous domain is colored red. The other two polymers have equal volume fractions in this case, but since $\chi_{\text {PMMA-P4VP }} \mathrm{N}$ is much less than $\chi_{\mathrm{PS}-\mathrm{P} 4 \mathrm{VP}} \mathrm{N}$, we conclude that the central cylinders consist of PS (green) surrounded by rings of PMMA (blue). Identification of the different domains in other AFM phase images proceeded in a similar manner.

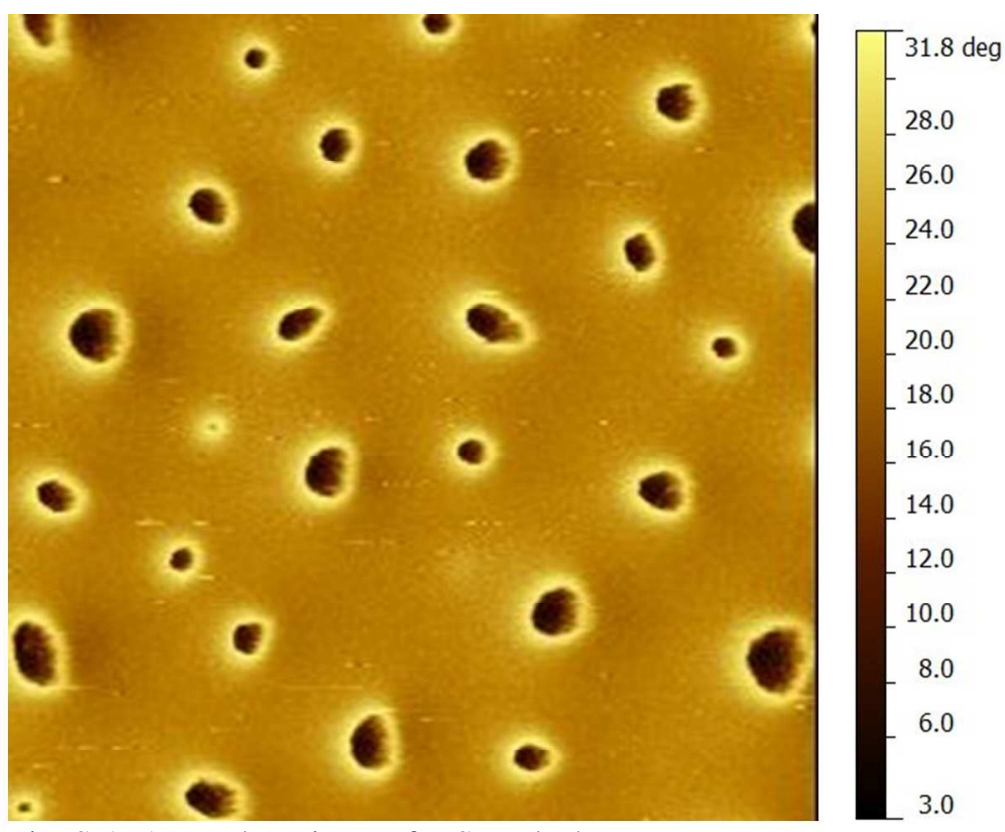

Fig. SI1: AFM phase image for Sample 4B. 


\section{Self-Consistent Field Simulations}

The phase behavior and morphologies of ternary brushes in the melt state were calculated using an extension to three species of a field theoretic model introduced in our previous work on binary melt brushes. ${ }^{1,5}$ The polymers are modeled as continuous Gaussian chains tethered to the substrate, which is modeled as a hard, impenetrable wall. We assume that the free surface is neutral to all three polymers and that there is a large polymer-air surface tension, and so we also treat the free surface as a flat, hard wall. We consider monodisperse A, B, and C tethered homopolymers, with equal chain lengths $N$. Length scales in the simulation results are in units of $R_{g}$. Throughout this work, the ternary brush is assumed to have a height of $L_{z}=3 R_{g}$. Since the system is a melt, the height fixes the overall grafting density. Periodic boundary conditions are employed in the plane of the brush in the $x$ and $y$ directions.

We use a standard Gaussian-chain model with harmonic stretching energy for the chains. The interaction energies are modeled with Flory-type local contact interactions between A, B, and C polymer segments, denoted by the Flory parameters $\chi_{\mathrm{AB}}, \chi_{\mathrm{AC}}$, and $\chi_{\mathrm{BC}}$. In addition to the harmonic stretching energy of the Gaussian chain and the segmental interaction energy, a penalty for local density fluctuations away from the average density $\rho_{0}$ is modeled using the compressible Helfand model, characterized by a stiffness parameter $\zeta$. In all calculations we set $\zeta N=100$, so the brush is relatively incompressible. A mean-field approximation is used to approximate the partition function of the system and calculate the equilibrium free energy. The SCFT free energy functional for the ternary melt brush is

$$
\begin{gathered}
\beta F \propto \int_{V} d \boldsymbol{r}\left[\sum_{\alpha \neq \beta} \frac{1}{\chi_{\alpha \beta} N}\left(W_{\alpha \beta}^{-}(\boldsymbol{r})^{2}-\left(i W_{\alpha \beta}^{+}(\boldsymbol{r})\right)^{2}\right)-\frac{1}{2 \zeta N}\left(i W^{+}(\boldsymbol{r})\right)^{2}-i W^{+}(\boldsymbol{r}) \phi(\boldsymbol{r})\right] \\
-\sum_{\alpha} \sigma_{\alpha} \int d x_{\perp} \ln \mathcal{Q}_{\alpha}\left(x_{\perp} ;\left[W_{\alpha}\right]\right)
\end{gathered}
$$

Here $\beta=1 / k T$ is the inverse temperature, the $\mathcal{Q}_{\alpha}$ are single chain partition functions for each polymer $(\alpha=\mathrm{A}, \mathrm{B}, \mathrm{C})$, and $\phi(\boldsymbol{r})$ is the total polymer segment density in the brush. The potential fields $W_{\alpha}$ that act on each chain are defined by

$$
\begin{aligned}
& W_{A}=i\left(W^{+}+W_{A B}^{+}+W_{A C}^{+}\right)+W_{A B}^{-}+W_{A C}^{-} \\
& W_{B}=i\left(W^{+}+W_{A B}^{+}+W_{B C}^{+}\right)+W_{B C}^{-}-W_{A B}^{-} \\
& W_{C}=i\left(W^{+}+W_{A C}^{+}+W_{B C}^{+}\right)-W_{A C}^{-}-W_{B C}^{-}
\end{aligned}
$$

Here, $W^{+}$is a pressure-like field that couples to the total density fluctuations in the system. The $W_{\alpha \beta}^{-}$fields are exchange fields that couple to composition fluctuations described by $\rho_{\alpha \beta}^{-}=\rho_{\alpha}-$ $\rho_{\beta}$, while the $W_{\alpha \beta}^{+}$fields couple to $\rho_{\alpha \beta}^{+}=\rho_{\alpha}+\rho_{\beta}$, where the $\rho_{\alpha}$ are microscopic density operators for each monomer type $\alpha$. Each chain is grafted at a location $x_{\perp}$ in the plane of the substrate, while the brush extends in the $z$ direction. In this work we assume uniform grafting of each polymer, so the grafting density of each polymer is simply $\sigma_{\alpha}=V \bar{\phi} f_{\alpha}$, where $V$ is the volume of the system, $\bar{\phi}$ is the average monomer density, and $f_{\alpha}$ is the total fraction of polymer $\alpha$. Further details of the SCFT and its numerical implementation are the same as in our previous work. 
Two types of SCFT calculations were performed. To calculate the phase diagram, only one unit cell of the morphology of interest was included. The calculations were started with random initial field configurations, and relaxed to the SCFT solution using a simple steepest descent search method until the error was less than $10^{-6}$. The optimal lateral system size was determined by boxrelaxation, resulting in a system with negligible internal stress. ${ }^{5}$ The box dimensions in the $x$ and $y$ directions were either allowed to vary independently, or alternatively were constrained to have a ratio of $L_{y}=\sqrt{3} L_{x}$ in order to promote hexagonal phases. The phase diagram was constructed by comparing the free energies of the candidate phases. To compare morphologies to experimental AFM images, we performed large cell SCFT calculations in a box with $\mathrm{L}_{\mathrm{x}}=\mathrm{L}_{\mathrm{y}}=50 \mathrm{R}_{\mathrm{g}}$, with random initial field configurations. These calculations were immediately quenched to the final values of the $\chi_{\alpha \beta} N$. The convergence to the SCFT solution was slow, due to the formation of defects. In all cases, we ran field updates until the difference in free energy between two successive states was less than $0.00003 \mathrm{kT} / \mathrm{R}_{\mathrm{g}}{ }^{3}$. We did not find any phases in the large-cell simulations that were different from those found in the unit cell calculations.
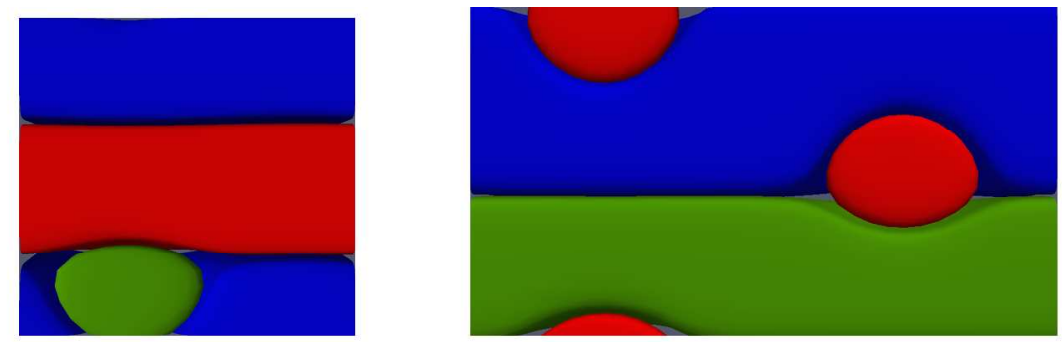

Fig. SI2: Unit cells for other decorated ripple phases. Left: B-decorated ripple, at $f_{A}=0.5, f_{B}=$ $0.1, \mathrm{f}_{\mathrm{C}}=0.4$; Right: C-decorated ripple, at $\mathrm{f}_{\mathrm{A}}=0.5, \mathrm{f}_{\mathrm{B}}=0.4, \mathrm{f}_{\mathrm{C}}=0.1$.

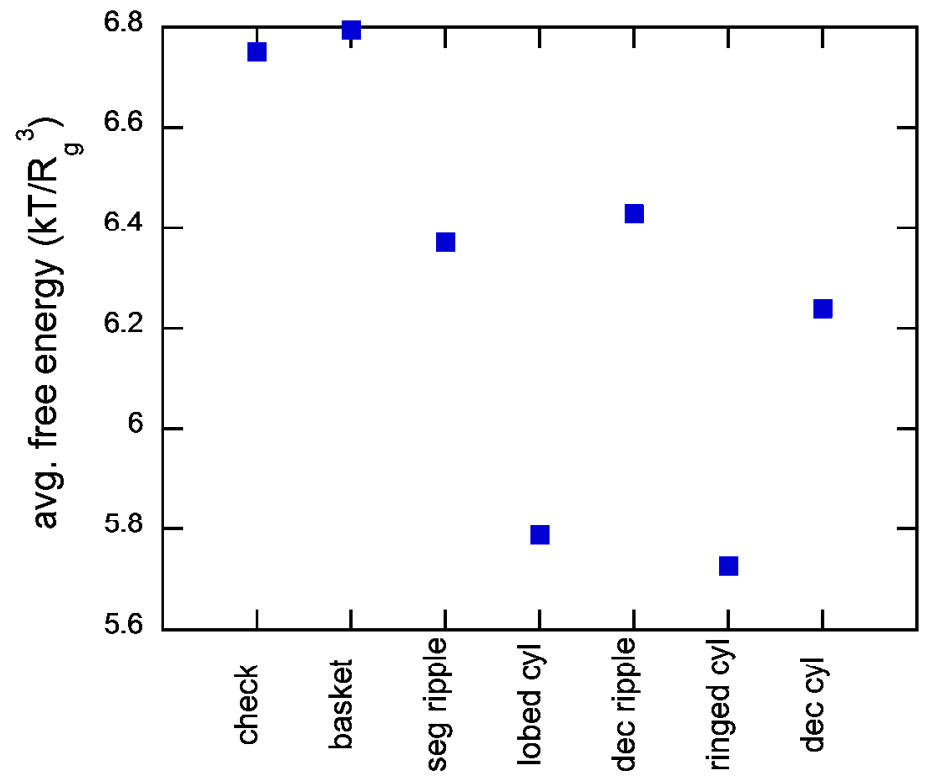

Figure SI3: Average free energy density for the seven phases found from the unit cell calculations using SCFT. 


\section{References}

(1) Price, A. D.; Hur, S.-M.; Fredrickson, G. H.; Frischknecht, A. L.; Huber, D. L. Macromolecules 2012, 45 (1), 510-524.

(2) Brandrup, J.; Immergut, E. H.; Grulke, E. A. Polymer Handbook, 4th ed.; John Wiley \& Sons: New York, 1999.

(3) Chen, Z.; Cai, J. I. E.; Zhang, L.; Liu, Y. U. N.; Jiang, X.; Yang, C. 2004, 13321343.

(4) Lemieux, M.; Usov, D.; Minko, S.; Stamm, M.; Shulha, H.; Tsukruk, V. V. Macromolecules 2003, 7244-7255.

(5) Hur, S.-M.; Frischknecht, A. L.; Huber, D. L.; Fredrickson, G. H. Soft Matter 2011, 7 (19), 8776. 